\title{
Local realizations of contact interactions in two- and three-body problems
}

\author{
A.T. Kruppa ${ }^{1}$, K. Varga ${ }^{1,2}$ and J. Revai ${ }^{3}$ \\ ${ }^{1}$ Institute of Nuclear Research of the Hungarian Academy of Sciences, H-4001 Debrecen, Pf. 51, \\ Hungary \\ ${ }^{2}$ Physics Division, Argonne National Laboratory, Argonne, Illinois 60439, USA \\ ${ }^{3}$ KFKI Research Institute for Particle and Nuclear Physics, H-1525, Budapest, P.O.B. 49, \\ Hungary
}

\begin{abstract}
Mathematically rigorous theory of the two-body contact interaction in three dimension is reviewed. Local potential realizations of this proper contact interaction are given in terms of Pöschl-Teller, exponential and square-well potentials. Three body calculation is carried out for the halo nucleus ${ }^{11} \mathrm{Li}$ using adequately represented contact interaction.
\end{abstract}

PACS numbers: 21.45.+v, 21.60-n, 21.30.-x, 27.20.+n 


\section{INTRODUCTION}

The contact interaction, or as sometimes called point or zero range interaction, plays an important role in nuclear physics. The short range nucleon-nucleon interaction is often simulated by a contact interaction in different models. An effective interaction of contact type (Skyrme-force) has been very frequently and successfully used in mean-field descriptions of heavy nuclei including the Hartree-Fock or Hartree-Fock-Bogoliubov theory (see e.g. [1]). Recently contact interaction has been applied in three-body calculations of halo nuclei [2 [4] and in the study of the role of resonance states in pair correlations [5]. Another example is the constituent-quark model for the application of the contact interaction. Several nonrelativistic three-body calculations have calculated the spectra of the three quark system [6] 8]. Despite the popularity of the contact force even its definition is problematic. The Hamiltonian of a two-body system interacting via a contact interaction is written as

$$
\hat{H}_{\delta}=-\frac{\hbar^{2}}{2 \mu} \Delta_{\mathbf{r}}+\lambda \delta(\mathbf{r})
$$

where $\mu$ is the reduced mass, $\Delta_{\mathbf{r}}$ is the three dimensional Laplacian, $\delta(\mathbf{r})$ is the Dirac delta-function and $\lambda$ is the "strength" of the contact interaction. The interpretation of the equation (1) is not trivial due to the singular nature of the Dirac delta-function. By using a complete set of states to diagonalize the above Hamiltonian one gets infinite binding energy for the system. To get a more meaningful result one usually truncates the configuration space [2 5,8] with some "reguralization" scheme. This truncation leads to a model dependence and results of different approaches can only be related and reproduced if one uses the same strategy to reguralize the interaction. Due to this approach it is widely believed that the contact interaction is meaningful only in a truncated space.

In this paper we propose a different approach where the application of the contact interaction is based on mathematically rigorous theory [9]. This proper contact interaction (PCI) is free from the above mentioned problems. The PCI gives finite energy and there is no dependence on basis truncations or extra parameters. This PCI has zero range and it 
acts non-trivially only in the $l=0$ partial wave. These two properties are what physicists expect from a contact interaction.

The contact force was introduced in nuclear physics by Bethe and Peierls [10] and Thomas [11] in 1935. The mathematically correct treatment of the operator (11) dates back to the work of Berezin and Faddeev [12] in 1961. An English review of the early Russian works on two and three-body problems with PCI can be found in Ref. [13]. Mathematically rigorous treatment of the operator (価) is given by different approaches. One of them is the coupling constant re-normalization procedure of Berezin and Faddeev [12]. This technique was applied also in two dimensions [14. Other approaches are the reguralization of the Green-function [15,16], the dimensional reguralization [17], or the theory of the Dirichlet forms [18]. We will use the self-adjoint extension theory of the contact force which is described in great detail in the monograph [9]. Each of these approaches leads to the same spectrum of the two-body Hamiltonian with PCI.

The mathematically correct formulation of the PCI in two-body problem is completely solved. It gives the two-body t-matrix in analytical form. This t-matrix can be used in subsequent three-body calculations based on the Faddeev equations. However, if we want to solve the three-body Schrödinger equation in coordinate space using some approximations then we need a local realization of the PCI. The aim of the paper is to give a few explicit local potential realizations of the PCI and apply it in three-body calculations.

The plan of the paper is the following. In section II the two-body spectrum of the PCI is discussed. In part III the general theory of the local potential realization of the PCI is reviewed and a few explicit realizations are given. In sections II and III.A those theorems of the monograph 9] are reviewed which are relevant for our purpose. In section IV the numerical solution of the two-body problem with PCI are shown. Finally, at the section V we present three-body calculation with PCI for the halo nuclei ${ }^{11} \mathrm{Li}$. 


\section{CONTACT INTERACTION IN TWO-BODY PROBLEM}

Our review of the mathematically correct treatment of the contact interaction is based on the monograph of Albeverio et al. [9]. The self-adjoint extension approach can be originated from the intuitive picture of the Dirac delta-function. The "function" $\delta(\mathbf{r})$ is zero if $\mathbf{r}$ is different from the zero vector $\mathbf{0}$. This means that the "operator" $\hat{H}_{\delta}$ is "identical" with the

kinetic energy $-\frac{\hbar^{2}}{2 \mu} \Delta_{\mathbf{r}}$ if $\mathbf{r} \neq \mathbf{0}$. In mathematical terms this means that the operator $-\frac{\hbar^{2}}{2 \mu} \Delta_{\mathbf{r}}$ acts only on the functions which belong to the function space $C_{0}^{\infty}\left(\mathcal{R}^{3} \backslash \mathbf{0}\right)$. This restricted Laplacian is not self-adjoint but its symmetric closure has many self-adjoint extensions. The self-adjoint extensions are characterized by a real number $\alpha(-\infty<\alpha<\infty)$. This self-adjoint extension, $\hat{H}_{\alpha, \delta}$, will give the proper representation of the contact interaction.

The spectrum of the operator $\hat{H}_{\alpha, \delta}$ is the following. The continuous spectrum is $[0, \infty)$ for each $\alpha$. If $\alpha<0$ then the $l=0$ partial wave component of $\hat{H}_{\alpha, \delta}$ has one bound state with energy

$$
E_{\alpha, \delta}=-(4 \pi \alpha)^{2} \frac{\hbar^{2}}{2 \mu} .
$$

The Hamiltonian $\hat{H}_{\alpha, \delta}$ has no bound states for higher partial waves. If the parameter of the self-adjoint extension is non-negative then there is no bound state in any partial wave but there is one virtual state in the partial wave $l=0$ with energy given by Eq. (2)).

The continuous spectrum may be characterized by the phase shift $\delta_{l}^{\alpha, \delta}(k)$, where $k$ denotes the wave number $k=\left(2 \mu E / \hbar^{2}\right)^{1 / 2}$ and $E>0$. In the partial wave $l=0$ the phase shift reads

$$
k \cot \left(\delta_{0}^{\alpha, \delta}(k)\right)=-\frac{1}{a_{\alpha, \delta}}
$$

where

$$
a_{\alpha, \delta}=-\frac{1}{4 \pi \alpha}
$$

The higher partial wave phase shifts are identically zero. The S-matrix in the partial wave $l=0$ has the following form 


$$
S_{0}^{\alpha, \delta}(k)=\frac{4 \pi \alpha+i k}{4 \pi \alpha-i k}
$$

The full characterization of a Hamiltonian is given if we know its Green-function. The Green-function of PCI in coordinate space can be given in closed analytical form [9].

The effective range expansion of an ordinary local spherical potential is of the form $k^{2 l+1} \cot \delta_{l}(k)=-1 / a_{l}+r_{l} k^{2} / 2+\ldots$, where $a_{l}$ is the scattering length and $r_{l}$ is the effective

range. The comparison of Eq. (3) with this expansion shows that the operator $\hat{H}_{\alpha, \delta}$ contains an interaction with zero range. The self-adjoint extension parameter not only determines the binding energy (see Eq. (2) if $\alpha<0)$ ) but it is also connected to the scattering length by Eq. (4).

At first it may seem strange that continuously many PCI exist. One can understand this using the following arguments (see e.g chapter 14.1 in Ref. [19]). If a spherical potential vanishes for $r \geq r_{0}$ then the solution of the radial Scrödinger equation is determined by assigning a given value to the logarithmic derivative of the wave function at $r_{0}$. This boundary condition depends on the energy. If the range of the potential $r_{0}$ tends to zero (contact force) then the boundary condition parameter becomes independent of the energy and in this way we can assign any value to it. Thus continuously many zero range interactions can be defined. This approach is an another way to define the PCI.

To sum up, the two-body problem with Dirac delta-potential can be solved analytically in a mathematically rigorous way. Infinite binding energy due to the contact force does not occur. The mathematically correct treatment of the contact force always leads to a finite and well defined results.

\section{LOCAL POTENTIAL REALIZATION OF THE CONTACT FORCE}

The abstract PCI can be realized by an appropriate limit of local potentials. We have to construct this local representation in such a way that it corresponds to the self-adjoint extension theory. Naively taking a potential which resembles to a Dirac $\delta$ function will not work. 


\section{A. Realization of the contact interaction by local potential}

The recipe for local realization of the contact interaction is given in the monograph [9] (see theorem 1.2.5). Let us take any local potential $V(\mathbf{r})$ which is in the Rollnik class 9, 19] and assume that $\int d \mathbf{r}(1+r)|V(\mathbf{r})|$ is finite. We define a Hamiltonian family by

$$
\hat{H}_{\delta}(\epsilon)=-\frac{\hbar^{2}}{2 \mu} \Delta_{\mathbf{r}}+\frac{\lambda(\epsilon)}{\epsilon^{2}} V(\mathbf{r} / \epsilon),
$$

where $\epsilon>0$ and $\lambda(\epsilon)$ is an arbitrary analytic function of $\epsilon$ such that $\lambda(0)=1$. Let us assume further that the potential $V(\mathbf{r})$ has a non-degenerate zero energy resonance, i.e.

$$
-\frac{\hbar^{2}}{2 \mu} \Delta_{\mathbf{r}} \Phi+V(\mathbf{r}) \Phi=0,
$$

for some $\Phi$. Because $\Phi$ is a resonance state it is not square integrable function. The normalization is defined by the following condition

$$
\int d \mathbf{r} V(\mathbf{r})|\Phi(\mathbf{r})|^{2}=-1 .
$$

Under these circumstances the Green-operator of the Hamiltonian family (6) converges to

the Green-operator of $\hat{H}_{\alpha, \delta}$ in norm resolvent sense if $\epsilon \rightarrow 0$. The self-adjoint extension parameter is given by

$$
\alpha=-\lambda^{\prime}(0) \frac{\hbar^{2}}{2 \mu}\left(\int d \mathbf{r} V(\mathbf{r}) \Phi(\mathbf{r})\right)^{-2} .
$$

The theorem 1.2.5 of Ref. [9] includes other cases. Here we have quoted only the part that we will use.

\section{B. Realization by Pöschl-Teller potential}

We will realize the result of the previous section using the Pöschl-Teller potential. It is known that in this case the radial Scrödinger equation

$$
-\frac{\hbar^{2}}{2 \mu} u^{\prime \prime}(r)-\frac{\hbar^{2}}{2 \mu b^{2}} \frac{s(s+1)}{\cosh (r / b)^{2}} u(r)=E u(r)
$$


can be solved analytically [20]. The energy eigenvalues are given by

$$
E_{n}=-(s-1-2 n)^{2} \frac{\hbar^{2}}{2 \mu b^{2}},
$$

where $n=0,2,4 \ldots$, and $s$ is an arbitrary complex number. If we choose the strength so that $s=2 \nu+1$, where $\nu=0,2,4, \ldots$, then we will have solutions with zero energy for the state $n=\nu$. The corresponding wave functions are

$$
u_{\nu}(r) \propto C_{2 \nu+1}^{1 / 2}(\tanh (r / b))
$$

where $C_{2 \nu+1}^{1 / 2}$ is the Gegenbauer polynomial. It is easy to see that these solutions are not square integrable so we have potentials which have zero energy resonance states. For simplicity we will consider the case $\nu=0$. The $\Phi$ of Eq. (7) with the normalization (8) now reads

$$
\Phi(\mathbf{r})=\left(\frac{2 \mu b}{\hbar^{2}}\right)^{1 / 2} \frac{\sqrt{3} \tanh (r / b)}{r \sqrt{8 \pi}} .
$$

The self-adjoint extension parameter can be calculated using Eqs. (9) and (13),

$$
\alpha=-\frac{\lambda^{\prime}(0)}{6 b \pi}
$$

Let us introduce the Hamiltonian family corresponding to Eq. (6),

$$
\hat{H}_{P T}(\epsilon)=-\frac{\hbar^{2}}{2 \mu} \Delta_{\mathbf{r}}-\frac{\lambda(\epsilon)}{b^{2} \epsilon^{2}} \frac{\hbar^{2}}{2 \mu} \frac{2}{\cosh ^{2}(r /(b \epsilon))} .
$$

We will call this operator the scaled Pöschl-Teller realization of the PCI. According to the previous section the resolvent of (15) converges to the resolvent of $\hat{H}_{\alpha, \delta}$ as $\epsilon \rightarrow 0$. We thus have a local realization of the PCI.

In this example the convergence of the Hamiltonian family (15) in the limit $\epsilon \rightarrow 0$ can be easily shown using elementary calculations. Since the eigenvalue problem of the scaled Pöschl-Teller operator (15) can be written in the form (10) the eigenvalues of (15) read

$$
E_{P T}(\epsilon, n)=-\left(\frac{s(\epsilon)-1-2 n}{b \epsilon}\right)^{2} \frac{\hbar^{2}}{2 \mu}
$$


where

$$
s(\epsilon)=\frac{1}{2}\left[-1+(1+8 \lambda(\epsilon))^{1 / 2}\right]
$$

and $n=0,2,4, \ldots$. Let us calculate $E_{P T}(\epsilon, n=0)$ in the limit $\epsilon \rightarrow 0$. We get

$$
\lim _{\epsilon \rightarrow 0} E_{P T}(\epsilon, 0)=\lim _{\epsilon \rightarrow 0}-\left(\frac{s(\epsilon)-1}{\epsilon}\right)^{2} \frac{\hbar^{2}}{2 \mu b^{2}}=-s^{\prime}(0)^{2} \frac{\hbar^{2}}{2 \mu b^{2}},
$$

since in the limit $\epsilon \rightarrow 0$ we have $s(\epsilon) \rightarrow 1$. Calculating the derivative of $s(\epsilon)$ at $\epsilon=0$ we end up with

$$
\lim _{\epsilon \rightarrow 0} E_{P T}(\epsilon, 0)=-\frac{4}{9} \lambda^{\prime}(0)^{2} \frac{\hbar^{2}}{2 \mu b^{2}}
$$

Thus we get the same energy as in the abstract theory of the PCI, i.e., if we substitute (14) into (2).

The $s$-wave S-matrix of the scaled Pöschl-Teller potential is of the following form [20]

$$
S_{0}^{P T}(k, \epsilon)=-\left(\frac{1}{2}\right)^{2 i k b \epsilon} \frac{\Gamma(i k b \epsilon) \Gamma\left(\frac{1}{2}(2+s(\epsilon)-i k b \epsilon)\right) \Gamma\left(\frac{1}{2}(1-s(\epsilon)-i k b \epsilon)\right)}{\Gamma(-i k b \epsilon) \Gamma\left(\frac{1}{2}(2+s(\epsilon)+i k b \epsilon)\right) \Gamma\left(\frac{1}{2}(1-s(\epsilon)+i k b \epsilon)\right)} .
$$

Using the identity $\Gamma(1+z)=z \Gamma(z)$ of the Gamma function we can easily calculate the limit $\epsilon \rightarrow 0$ of the S-matrix $(20)$

$$
\lim _{\epsilon \rightarrow 0} S_{0}^{P T}(k, \epsilon)=\lim _{\epsilon \rightarrow 0} \frac{1-s(\epsilon)+i k b \epsilon}{1-s(\epsilon)-i k b \epsilon}=\frac{s^{\prime}(0)+i k b}{-s^{\prime}(0)-i k b} .
$$

Taking into account Eq. (14) and $s^{\prime}(0)=2 \lambda^{\prime}(0) / 3$ we see that the limit agrees with the expression (5) given by the general abstract theory of the PCI.

\section{Realization by exponential and square well potentials}

A local realization of the abstract result can be given not only with the Pöschl-Teller potential but by any local potential satisfying the conditions of section III.A. Here we take first the exponential potential as an another example. The eigenenergies of the radial Scrödinger equation, 


$$
-\frac{\hbar^{2}}{2 \mu} u^{\prime \prime}(r)-V_{0} \exp (-r / b) u(r)=E u(r)
$$

can be obtained from the transcendental equation [19

$$
J_{2 i b k}\left(2 b \sqrt{\frac{2 \mu V_{0}}{\hbar^{2}}}\right)=0
$$

where $J_{\nu}$ is the Bessel function. The condition of the zero energy resonance reads

$$
J_{0}\left(2 b \sqrt{\frac{2 \mu V_{0}}{\hbar^{2}}}\right)=0
$$

This equation defines the strength of the potential. The corresponding resonance wave function with the normalization given in section III.A is

$$
\Phi(\mathbf{r})=\frac{1}{r} \frac{1}{\sqrt{4 \pi b V_{0}} J_{1}\left(b_{0}\right)} J_{0}\left(b_{0} \exp (-r / 2 b)\right)
$$

where $b_{0}=2 b\left(2 \mu V_{0} / \hbar^{2}\right)^{1 / 2}$.

The scaled Hamiltonian family which converges to the PCI in the limit $\epsilon \rightarrow 0$ is

$$
-\frac{\hbar^{2}}{2 \mu} \Delta_{\mathbf{r}}-V_{0} \frac{\lambda(\epsilon)}{\epsilon^{2}} \exp (-r / b \epsilon)
$$

Note that the potential strength $V_{0}$ is determined by Eq. (24). We call the operator (26) the scaled exponential realization of the PCI. The self-adjoint extension parameter can be calculated using Eqs. (9) and (25)

$$
\alpha=-\lambda^{\prime}(0) \frac{\hbar^{2}}{2 \mu} \frac{b J_{1}\left(b_{0}\right)^{2}}{4 \pi V_{0}}\left[\int_{0}^{\infty} d r r \exp (-r / b) J_{0}\left(b_{0} \exp (-r / 2 b)\right)\right]^{-2} \text {. }
$$

The integral in (27) can be calculated numerically.

The abstract PCI can also be realized by a properly chosen square well potential. This latter is defined in the usual way: $V^{S Q}(r)=-V_{0}$ if $r<R$ and $V^{S Q}(r)=0$ if $r>R$. The condition of the presence of a resonance with zero energy is that the strength of the square well is

$$
V_{0}=\frac{\hbar^{2}}{2 \mu}\left[\frac{(2 k+1) \pi}{R}\right]^{2}
$$


where $k=0, \pm 1, \pm 2, \ldots$. The properly normalized zero energy resonance wave function reads

$$
\Phi(\mathbf{r})=\frac{1}{r}\left(2 \pi V_{0} R\right)^{-1 / 2} \sin \left(\sqrt{\frac{2 \mu}{\hbar^{2}} V_{0} r}\right) .
$$

The self-adjoint extension parameter is given by

$$
\alpha=-\lambda^{\prime}(0) \frac{2 \mu}{\hbar^{2}} \frac{R V_{0}}{8 \pi}
$$

The Hamiltonian family which tends to the PCI is the following

$$
-\frac{\hbar^{2}}{2 \mu} \Delta_{\mathbf{r}}+\frac{\lambda(\epsilon)}{\epsilon^{2}} \hat{V}_{\epsilon}^{S Q}(r)
$$

where $V_{\epsilon}^{S Q}(r)=-V_{0}$ if $r<R \epsilon$ and $V_{\epsilon}^{S Q}(r)=0$ if $r>R \epsilon$.

\section{NUMERICAL CALCULATION: TWO-BODY PROBLEM}

We have seen that the eigenvalue problem of the Pöschl-Teller realization of the PCI can be solved exactly for any $\epsilon$. Since we will solve the three-body problem numerically, using the stochastic variational method [22], it is essential to see how this numerical method performs for the solution of Eq. (15) or any other local realization of the abstract PCI. This numerical exercise is indeed a new test for the stochastic variational method since the potential of (15) really resembles the Dirac delta-function. A few scaled Pöschl-Teller potentials are shown in Fig. 1. for $b=1 \mathrm{fm}$.

For the function $\lambda(\epsilon)$ we have chosen the following ansatz $\lambda(\epsilon)=1+C \epsilon$. With the parameter $C$ we can change $\lambda^{\prime}(0)$ which influences the value of the self-adjoint extension parameter. This latter uniquely determines the bound state energy and the scattering length of the PCI.

For the two body problem we considered the bound neutron-proton system and the scattering states of the neutron-neutron system. For the deuteron case the exact eigenvalues and the numerically calculated ones using the scaled Pöschl-Teller potential are compared in Table I for several values of the scaling parameter $\epsilon$. We have used $\hbar^{2} /(2 \mu)=41.47 \mathrm{MeV} \mathrm{fm}^{2}$ 
in all calculations. Table I demonstrates that the stochastic variational method is able to deal with such an extreme potential. Of course, numerically, we could not carry out the limit $\epsilon \rightarrow 0$. Using the reliable results of the stochastic variational method $(\epsilon \leq 0.005)$ the numerically calculated values can be extrapolated to $\epsilon=0$. From Table I we can conclude that the calculation with $\epsilon=0.005$ is very satisfactory; the error is less than $10 \mathrm{eV}$. Having this experience, in three-body calculations we will accept the result of the calculation with scaling $\epsilon=0.005$ (or the extrapolated value to $\epsilon=0$ ) as the result of the PCI.

The numerical results of the exponential and square well realization of the PCI are given in Table II. The parameters of the potentials were $V_{0}=0.936831 \mathrm{MeV}, b=8 \mathrm{fm}$ and $V_{0}=4.092925 \mathrm{MeV}$ and $R=5 \mathrm{fm}$ in the case of the exponential and square well potentials, respectively. The stochastic variational calculation performs very well for the exponential potential. It was not possible get better accuracy than four digits in the case of a square well potential. The convergence of the exponential and square well realization to the PCI is not as quick as in the case of the Pöschl-Teller realization.

The phase shift of the scaled Pöschl-Teller potential $\delta_{0}^{P T}(k, \epsilon)$ can be deduced from Eq. (20). The quantity $k \cot \delta_{0}^{P T}(k, \epsilon)$ is displayed in Fig. 2 as the function of the wave number for several values of the scaling parameter. We see that decreasing the scaling parameter the result converges to the phase shift of the PCI. The self-adjoint extension parameter of the PCI in this calculation is fixed to give the experimentally observed scattering length $(-18.5 \mathrm{fm})$ of the dineutron. In order to get the effective range parameters of the scaled Pöschl-Teller potential we make the expansion:

$$
k \cot \delta_{0}^{P T}(k, \epsilon)=i k\left(S_{0}^{P T}(k, \epsilon)+1\right) /\left(S_{0}^{P T}(k, \epsilon)-1\right) \approx-1 / a_{n n}+r_{0}^{2} k^{2} / 2 .
$$

At each scaling the scattering length and the effective range obtained in this way are shown in Tables I and III for the scaled Pöschl-Teller potentials.

To sum up, in numerical calculations we can only decrease the effective range to $0.01 \mathrm{fm}$ but this is already enough to get the binding energy with an accuracy less than $10 \mathrm{keV}$. 


\section{CONTACT INTERACTION IN THREE-BODY PROBLEM}

We have seen that the contact interaction is very tricky even for the two-body problem. Further complications can be expected when three particles interact. If each pair interaction is a contact force then the three-body problem can be solved exactly [12, 13]. The final result is, however, physically unsatisfactory due to the Thomas effect: there is an infinite number of bound states and the spectrum is not bounded from below [11,21. We mention that in this three-body problem the self-adjoint extension type approach have to be used to have a meaningful three-body Hamiltonian.

In the present paper we consider a three-body system where only one pair of the particles interacts through a contact force and the other two pair interactions are given by short range local potentials. The physical motivation of such a three-body system is to model nuclei

with halos, like ${ }^{6} \mathrm{He}$ or ${ }^{11} \mathrm{Li}$. The situation in this case is simpler. If we want to include the PCI in this type of three-body problem we can proceed in two different ways.

For the solution of the three-body problem we may use the Faddeev theory. In this case one needs the Green-operator of each subsystem of the three particles. Since one pair of particles interact via PCI the Green-operator of this two body subsystem is known exactly and analytically. This property leads to a simplification of the Faddeev equations. It can be shown that this type of three-body problem is well defined and physically meaningful. The theoretical framework of this Faddeev type approach was developed in 23].

If we want to solve the three-body problem using the Scrödinger equation in coordinate space then we are ready to use the PCI since we have several local realizations of the PCI. Since we are interested in bound state properties of three-body systems we can use the Rayleigh-Ritz variational principle to calculate the binding energies. For the three body problem we have chosen a simple model of the light halo nucleus ${ }^{11} \mathrm{Li}$ which presently is in the focus of many theoretical and experimental investigations.

Let us consider a simple model: an inert core and two neutrons. The three-body Hamiltonian using Jacobi coordinates has the form 


$$
-\frac{\hbar^{2}}{2 \mu} \Delta_{\mathbf{r}}-\frac{\hbar^{2}}{2 M} \Delta_{\mathbf{R}}+V_{n, n}(\mathbf{r})+V_{n, c}(\mathbf{r}, \mathbf{R})
$$

Here $\mathbf{r}=\mathbf{r}_{\mathbf{1}}-\mathbf{r}_{\mathbf{2}}$ is the relative coordinate of the two neutrons and $\mathbf{R}=\frac{1}{2}\left(\mathbf{r}_{\mathbf{1}}+\mathbf{r}_{\mathbf{2}}\right)-\mathbf{r}_{\mathbf{3}}$ is the vector which points from the third particle (core) to the center of mass of the dineutron. As in Refs. [3, 1] we assume that the neutron-neutron interaction is a zero range contact force. However, we will not restrict the model space, as it was done in Refs. [3, [4], in order to get finite energy but we will use the PCI. For the neutron-neutron interaction we will take the scaled Pöschl-Teller and exponential realizations of the PCI. A shallow neutron core interaction is taken in order to avoid the problem of the Pauli projection. The explicit form of this potential is given in Ref. «1.

In Table IV two calculations with the Pöschl-Teller realization of the PCI are shown. In the first case the parameterization of the PCI corresponds to the experimental value $(-18.5$ $\mathrm{fm}$ ) of the scattering length of the free neutron-neutron system. The second calculation corresponds to the case when the parameter of the PCI is adjusted until the three-body energy reproduces the experimental value $\left(S_{2 n}=-0.295 \pm 0.035 \mathrm{MeV}\right)$ of the two-neutron separation energy of ${ }^{11} \mathrm{Li}$ [24]. This parameter of the PCI leads to a $-7.32 \mathrm{fm}$ scattering length for the dineutron. The mean square radius of the neutron-neutron and the coredineutron system is also calculated in this case.

From Table IV we can conclude that the effective neutron-neutron interaction is not consistent with the free neutron-neutron scattering. We had to reduce the scattering length of the dineutron system in order to reproduce the experimental value of the two neutron separation energy. Similar phenomena were observed in the calculations of Refs. [2 《4 where the traditional notion of the contact force was applied. The results of Table IV are very similar to the results of Ref. [4] where the standard method (restricted model space) was used to handle the contact interaction. Although the results are qualitatively similar there is a deep conceptual difference between the two calculations. In our calculations with PCI we indeed used a zero range force whereas in the works [3, 1], due to the restriction of the model space, the effective range is much larger than ours. In principle the PCI has zero 
range, in practice we can reach $0.01 \mathrm{fm}$. In the calculations of Ref. [3, 10 the effective range is roughly $2 \mathrm{fm}$ and this explain the quantitative difference of the two calculations.

We have repeated the three-body calculation, replacing the Pöschl-Teller realization by the exponential realization of the PCI. The results are shown in Table V. Of course, due to the different realization of the PCI, the energy corresponding to a given $\epsilon$ are different in Table $\mathrm{V}$ and in the second column of Table IV. What is important is that the extrapolated values, which correspond to the PCI, are very close to each other in both realizations.

Because we use the local realization of the PCI we have to carry out the limit $\epsilon \rightarrow$ 0 numerically in the three-body calculation and this requires great care. The stochastic variational method [22], which proved to be very accurate in few body problems, is used to solve the bound state three-body problem. It is clear from Tables IV and V that convergence is reached in the order of a few tenth $\mathrm{keV}$. This means that our proposed method for the correct handling of the contact interaction works for three-body problems.

\section{SUMMARY}

We have reviewed the mathematically, rigorously defined proper contact interaction and shown that it has the properties of the standard notion of the contact interaction. This proper contact interaction is free from the problem (infinite binding energy) of the standard treatment of the contact interaction. Local potential realizations of the abstract proper contact interaction were given in terms of Pöschl-Teller, exponential and square-well potentials.

The calculation for ${ }^{11} \mathrm{Li}$ revealed that the proper contact interaction with local realization can be incorporated into three-body calculations based on the coordinate space Schrödinger equation provided this latter is solved accurately enough.

\section{ACKNOWLEDGMENTS}

The work of K. V. was supported by the U. S. Department of Energy, Nuclear Physics Division, under contract No. W-31-109-ENG-39 and this research was also supported by 
Hungarian OTKA grants No. T029003 and T026244. 


\section{REFERENCES}

[1] J. Dobaczewski et al., Phys. Rev. C 53, 2809 (1996).

[2] G.F. Bertsch and H. Esbensen, Ann. Phys. 209, 327 (1991).

[3] H. Esbensen, G.F. Bertsch, Nucl.Phys. A542, 310 (1992).

[4] H. Esbensen, G.F. Bertsch and K. Hencken, Phys. Rev. C 56, 3054 (1997).

[5] J.R. Bennett, J. Engel and S. Pittel, Phys. Lett. B368, 7 (1996).

[6] L.Ya. Glozman, Z. Papp and W. Plessas, Phys. Lett. 381, 311 (1996).

[7] L.Ya. Glozman, Z. Papp,W. Plessas, K. Varga and R.F. Wagenbrunn, Phys. Rev. C 57,3406 (1998).

[8] F. Fernández, P. González and A. Valcarce, Few-Body Syst. Suppl. 10, 385 (1998).

[9] S. Albeverio, F. Gesztesy, R. Høegh-Krohn and H. Holden, Solvable Models in Quantum Mechanics (Springer-Verlag, 1988).

[10] H. Bethe and R. Peierls, Proc. Roy. Soc. (London) 148A, 146 (1935).

[11] L.H. Thomas, Phys. Rev. 47, 903 (1935).

[12] F.A. Berezin and L.D. Faddeev, Soviet. Math. Dokl. 2, 372 (1961).

[13] G. Flamand, Cargese Lectures in Theoretical Physics 1967, ed. by F.Lurcat (Gordon and Breach, New York, 1967),p. 247.

[14] C. Manuel and R. Tarrach, Phys. Lett. B328, 113 (1994).

[15] C. Grosche, Ann. Physik 3, 283 (1994).

[16] D.K. Park, J. Math. Phys. 36, 5453 (1995).

[17] I. Mitra, A.DasGupta and B. Dutta-Roy, Am. J. Phy. 66, 1101 (1998).

[18] S. Albeverio, R. Høegh-Krohn and L. Streit, J. Math. Phys. 18, 907 (1980). 
[19] R.G. Newton, Scattering Theory of Waves and Particles (Springer-Verlag, 1982).

[20] D. Baye, G.Lévai and J.-M. Sparenberg, Nucl. Phys. A599, 435 (1996).

[21] S.K. Adhikari at al., Phys. Rev. C 37, 3666 (1988).

[22] Y. Suzuki and K. Varga, Stohastic Variational Approach to Quantum-Mechanical FewBody Problems (Springer-Verlag, 1998).

[23] A.I. Baz, Ya. B. Zeldovich and A.M. Perelomov, Scattering, Reactions and Decay in Nonrelativistic Quantum Mechanics in Russian (Izdatelstvo Nauka, Moskva 1971).

[24] B.M. Yong et al., Phys. Rev. Lett. 71, 4124 (1993). 


\section{TABLES}

TABLE I. The binding energy in $\mathrm{MeV}$ of the deuteron in scaled Pöschl-Teller potential as the function of the scaling parameter. The exact result and the numerical one calculated by the stochastic variational method are shown. The binding energy of the proper contact interaction is 2.222496 MeV this corresponds to the limit $\epsilon \rightarrow 0$. The effective range $r_{0}$ of the scaled Pöschl-Teller potentials are also given.

\begin{tabular}{cccc}
\hline \hline$\epsilon$ & $r_{0}(f m)$ & $-E_{P T}$ numerical & $-E_{P T}$ exact \\
\hline 0.500 & 0.86 & 2.065933 & 2.065933 \\
0.100 & 0.19 & 2.188844 & 2.188843 \\
0.050 & 0.10 & 2.205511 & 2.205510 \\
0.010 & 0.02 & 2.219079 & 2.219073 \\
0.005 & 0.01 & 2.220789 & 2.220783 \\
\hline extrapolated to & & & \\
$\epsilon=0$ & 0.00 & 2.222502 & 2.222496 \\
\hline \hline
\end{tabular}


TABLE II. The binding energy in $\mathrm{MeV}$ of the deuteron in scaled exponential and square-well potentials as the function of the scaling parameter. The exact result and the numerical one calculated by the stochastic variational method are shown. The binding energy of the proper contact interaction is $2.222496 \mathrm{MeV}$ this corresponds to the limit $\epsilon \rightarrow 0$.

\begin{tabular}{ccccc}
\hline \hline & \multicolumn{2}{c}{ exponential } & \multicolumn{2}{c}{ square-well } \\
\hline$\epsilon$ & numerical & exact & numerical & exact \\
\hline 0.500 & 1.34894 & 1.34894 & 1.662 & 1.663 \\
0.100 & 1.93918 & 1.93919 & 2.077 & 2.080 \\
0.050 & 2.06796 & 2.06797 & 2.135 & 2.149 \\
0.010 & 2.18910 & 2.18909 & 2.196 & 2.207 \\
0.005 & 2.20562 & 2.20562 & 2.201 & 2.215 \\
\hline extrapolated to & & & & 2.222 \\
$\epsilon=0$ & 2.22250 & 2.22250 & 2.205 & \\
\hline \hline
\end{tabular}

TABLE III. The effective range parameters of the dineutron system in scaled Pöschl-Teller potential as the function of the scaling parameter. The effective range parameters of the proper contact interaction (scattering length $a_{n n}=-18.5 \mathrm{fm}$ and effective range $r_{0}=0 \mathrm{fm}$ ) corresponds to the limit $\epsilon \rightarrow 0$.

\begin{tabular}{cccccc}
\hline \hline$\epsilon$ & 0.500 & 0.1 & 0.05 & 0.01 & 0.005 \\
\hline$-a_{n n}(f m)$ & 19.85 & 18.38 & 18.43 & 18.49 & 18.49 \\
$r_{0}(f m)$ & 1.04 & 0.20 & 0.10 & 0.02 & 0.01 \\
\hline \hline
\end{tabular}


TABLE IV. Two neutron separation energy of ${ }^{11} \mathrm{Li}$ in the three-body model as the function of the scaling parameter of the Pöschl-Teller potential. The binding energy of the proper contact interaction can be obtained extrapolating to $\epsilon=0$. The self adjoint extension parameter of the proper contact interaction corresponds to the given neutron-neutron scattering length $a_{n n}$. The mean square neutron-neutron and core-dineutron radius are also given.

\begin{tabular}{c|c|ccc}
\hline \hline & $a_{n n}=-18.5 \mathrm{fm}$ & \multicolumn{3}{|c}{$a_{n n}=-7.32 \mathrm{fm}$} \\
\hline$\epsilon$ & $S_{2 n}(\mathrm{MeV})$ & $S_{2 n}(\mathrm{MeV})$ & $r_{n n}^{2}\left(\mathrm{fm}^{2}\right)$ & $r_{c n}^{2}\left(\mathrm{fm}^{2}\right)$ \\
\hline 0.500 & -0.5495 & -0.1590 & 86.3 & 113.0 \\
0.100 & -0.6949 & -0.2812 & 58.0 & 79.6 \\
0.050 & -0.7135 & -0.2975 & 55.1 & 76.0 \\
0.010 & -0.7268 & -0.3095 & 54.3 & 75.6 \\
0.005 & -0.7285 & -0.3112 & 54.1 & 75.3 \\
\hline extrapolated to & & & & 74.9 \\
$\epsilon=0$ & -0.7302 & -0.3130 & 53.9 & \\
\hline \hline
\end{tabular}


TABLE V. Two neutron separation energy of ${ }^{11} \mathrm{Li}$ in the three-body model as the function of the scaling parameter of the exponential potential. The binding energy of the proper contact interaction can be obtained extrapolating to $\epsilon=0$. The self adjoint extension parameter of the proper contact interaction corresponds to the free neutron-neutron scattering length $-18.5 \mathrm{fm}$.

\begin{tabular}{cc}
\hline \hline$\epsilon$ & $S_{2 n}(\mathrm{MeV})$ \\
\hline 0.100 & -0.2786 \\
0.050 & -0.4797 \\
0.010 & -0.6795 \\
0.005 & -0.7058 \\
0.0025 & -0.7188 \\
\hline extrapolated to & \\
$\epsilon=0$ & -0.7316 \\
\hline \hline
\end{tabular}




\section{FIGURES}

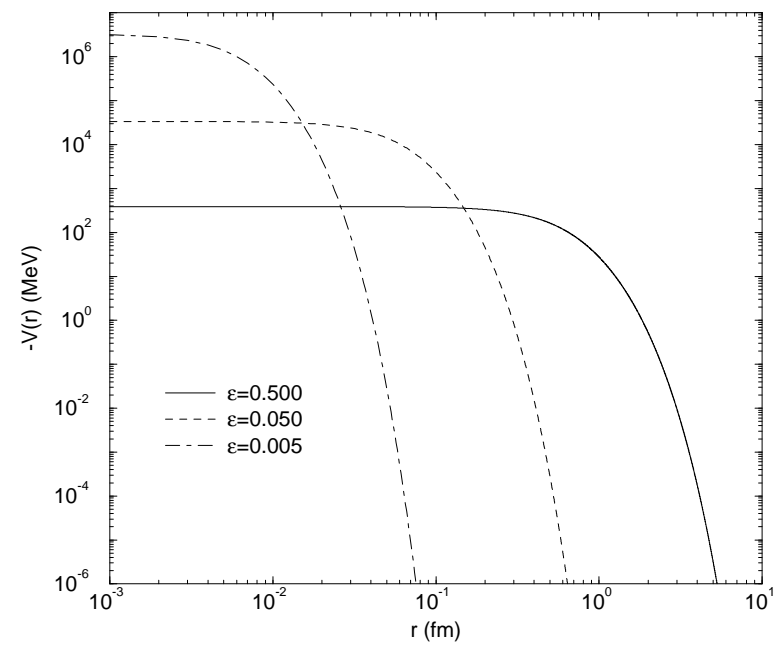

FIG. 1. Local potential realization of the proper contact interaction in the case of deuteron. Scaled Pöscl-Teller potentials with different scaling parameters. Note the logarithmic scale on each axis.

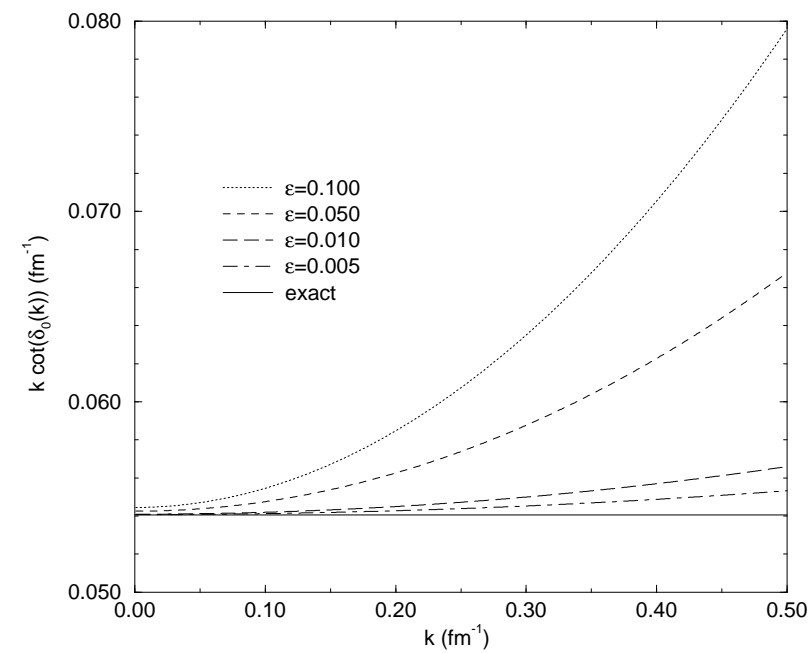

FIG. 2. The phase shift of the scaled Pöscl-Teller potential with different scaling parameters for the dineutron system. The parameter of the PCI corresponds to the experimental scattering length $a_{n n}=-18.5 \mathrm{fm}$. The quantity $k \cot \left(\delta_{0}(k)\right)$ is displayed. For the proper contact interaction this is independent of the wave number $k\left(k \cot \left(\delta_{0}(k)\right)=\frac{1}{18.5} \mathrm{fm}^{-1}\right)$ 